\title{
Quantification of myocardial late gadolinium enhancement using synthetic inversion recovery imaging
}

Akos Varga-Szemes ${ }^{1 *}$, Rob J van der Geest ${ }^{2}$, Bruce S Spottiswoode ${ }^{3}$, Giuseppe Muscogiuri ${ }^{1,4}$, Carlo N De Cecco ${ }^{1,4}$, Pal Suranyi ${ }^{1}$, Wolfgang $G$ Rehwald ${ }^{3,5}$, Joseph U Schoepf ${ }^{1}$

From 18th Annual SCMR Scientific Sessions

Nice, France. 4-7 February 2015

\section{Background}

Inversion recovery (IR) late gadolinium enhancement (LGE) cardiac magnetic resonance (CMR) is generally used for post-contrast infarct detection in the myocardium. Novel technological advancements allow for the generation of synthetic IR images based on the pixel-bypixel T1 values. Theoretically, synthetic IR images can be retrospectively generated at any inversion time (TI). In this study, we aimed to evaluate the diagnostic value of synthetic phase-sensitive IR (PSIR) images for the quantification of myocardial LGE.

\section{Methods}

The Institutional Review Board approved the study protocol. Consecutive patients $(n=31)$ underwent CMR using 1.5T Siemens MAGNETOM Avanto (Siemens AG, Erlangen, Germany) equipment. Conventional PSIR acquisition and fast T1-mapping (investigational prototype modified look-locker IR, MOLLI, 4(1)3(1)2 sampling scheme) of the heart were performed. This scheme acquires 9 images in 11 heartbeats with 3 inversions. For the MOLLI acquisition, an initial TI of $110 \mathrm{~ms}$ was used with a $80 \mathrm{~ms}$ TI increment. Synthetic PSIR images were

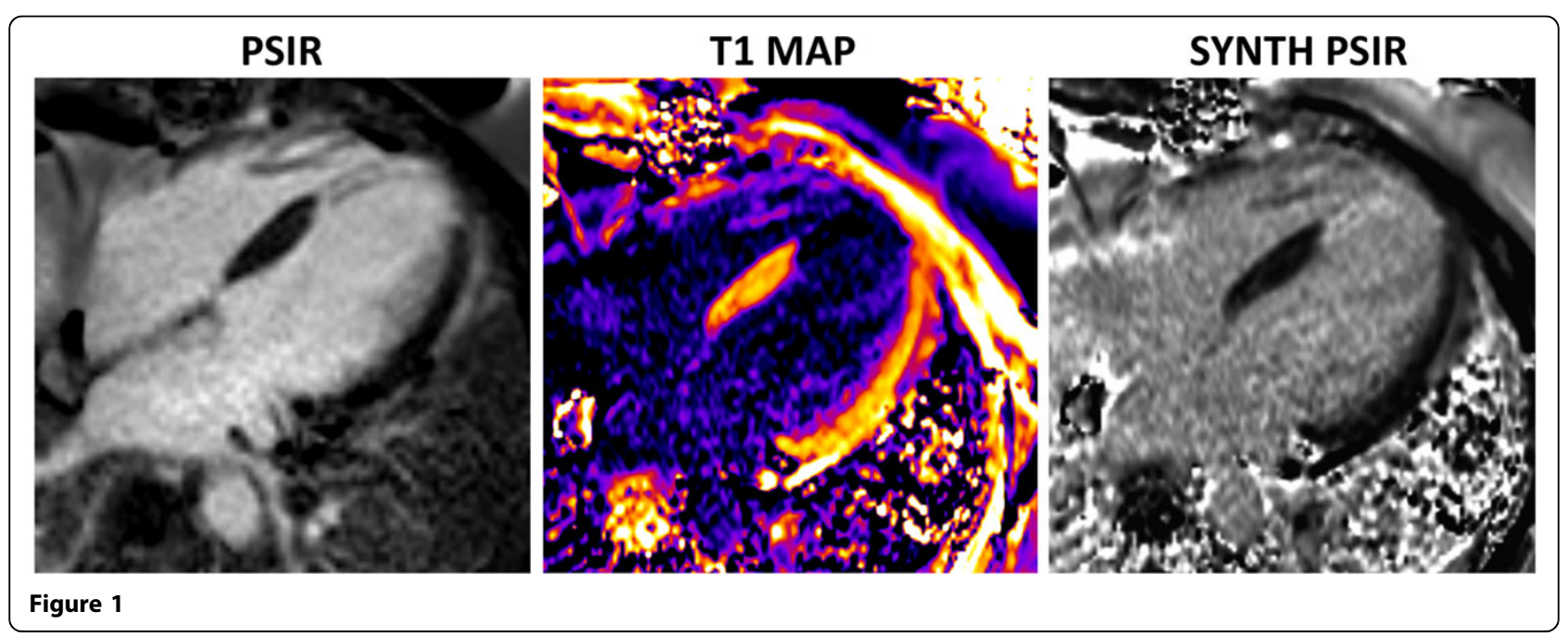

${ }^{1}$ Department of Radiology and Radiological Science, Medical University of

South Carolina, Charleston, SC, USA

Full list of author information is available at the end of the article 
calculated using an in-house developed, Microsoft Excel based application allowing the generation of these images at any TI. LGE was quantified by two independent readers employing a binary thresholding method using Research MASS analytical software. The accuracy of infarct quantification with the synthetic technique was compared to that of the conventional technique.

\section{Results}

LGE was observed in 11 (35.4\%) patients. LGE pattern was consistent with myocardial infarction in all cases. Representative conventional PSIR, as well as corresponding T1 map and synthetic PSIR images are shown in Figure 1. The infarct fraction measured by conventional and synthetic PSIR techniques was $16.5 \pm 7.4$ and $17.2 \pm 7.9 \%$, respectively, and statistically identical $(\mathrm{p}=0.238)$. Inter-observer studies revealed excellent agreement between the two readers (Kappa $>0.81$ ). There was excellent agreement in the detection of myocardial infarction between the two techniques.

\section{Conclusions}

In this project we have shown the feasibility of LGE detection and quantification using synthetic PSIR images. Synthetic images provide the same accuracy as the conventional PSIR images used in clinical practice as standard of care. With the increasing acceptance and availability of $\mathrm{T} 1$ mapping, the need for conventional PSIR images could be omitted in the future. Synthetic PSIR techniques would eliminate the need to optimize the LGE acquisition (TI scout, TI readjustment) without additional scanning time.

\section{Funding}

N/A.

\author{
Authors' details \\ 'Department of Radiology and Radiological Science, Medical University of \\ South Carolina, Charleston, SC, USA. ${ }^{2}$ Leiden University Medical Center, \\ Leiden, Netherlands. ${ }^{3}$ Siemens Medical Solutions, Chicago, IL, USA. \\ ${ }^{4}$ University of Rome "Sapienza", Rome, Italy. ${ }^{5}$ Duke Cardiovasular Magnetic \\ Resonance Center, Durham, NC, USA.
}

Published: 3 February 2015

doi:10.1186/1532-429X-17-S1-08

Cite this article as: Varga-Szemes et al:: Quantification of myocardial late gadolinium enhancement using synthetic inversion recovery imaging. Journal of Cardiovascular Magnetic Resonance 2015 17(Suppl 1):O8.
Submit your next manuscript to BioMed Central and take full advantage of:

- Convenient online submission

- Thorough peer review

- No space constraints or color figure charges

- Immediate publication on acceptance

- Inclusion in PubMed, CAS, Scopus and Google Scholar

- Research which is freely available for redistribution

Submit your manuscript at www.biomedcentral.com/submit
C Biomed Central 